ÄrZTINNEN FÜr FraUEN 
Ergebnisse der Frauenforschung

Band 39

Begründet und im Auftrag des Präsidenten der Freien Universität Berlin herausgegeben von

Prof. Anke Bennholdt-Thomsen, Germanistik

Elisabeth Böhmer, Soziologie

Prof. Marlis Dürkop, Sozialpädagogik

Prof. Ingeborg Falck, Medizin

Prof. Marion Klewitz, Geschichtsdidaktik

Prof. Jutta Limbach, Jura

Prof. Hans Oswald, Pädagogik

Prof. Renate Rott, Soziologie

Dr. Hanna Beate Schöpp-Schilling, Amerikanistik/Anglistik, Germanistik

Prof. Margarete Zimmermann, Romanistik

Koordination: Anita Runge 
Kristin Hoesch

\section{ÄRZTINNEN FÜR FRAUEN}

Kliniken in Berlin 1877-1914

Verlag J. B. Metzler

Stuttgart - Weimar 
Die Deutsche Bibliothek - CIP-Einheitsaufnahme

Hoesch, Kristin:

Ärztinnen für Frauen : Kliniken in Berlin 1877 - 1914 / Kristin Hoesch. - Stuttgart ; Weimar : Metzler, 1995

(Ergebnisse der Frauenforschung; Bd. 39)

Zugl.: Berlin, Freie Univ., Diss., 1994 u.d.T.: Hoesch, Kristin: Die Klinik und Poliklinik weiblicher Ärzte für Frauen in Berlin von 1877 bis 1914

ISBN 978-3-476-01347-7

ISBN 978-3-476-03626-1 (eBook)

DOI 10.1007/978-3-476-03626-1

NE: GT

Dieses Werk einschließlich aller seiner Teile ist urheberrechtlich geschützt. Jede Verwertung außerhalb der engen Grenzen des Urheberrechtsgesetzes ist ohne Zustimmung des Verlages unzulässig und strafbar. Das gilt insbesondere für Vervielfältigungen, Übersetzungen, Mikroverfilmungen und die Einspeicherung und Verarbeitung in elektronischen Systemen.

(C) 1995 Springer-Verlag GmbH Deutschland

Ursprünglich erschienen bei J. B. Metzlersche Verlagsbuchhandlung

und Carl Ernst Poeschel Verlag GmbH in Stuttgart 1995 


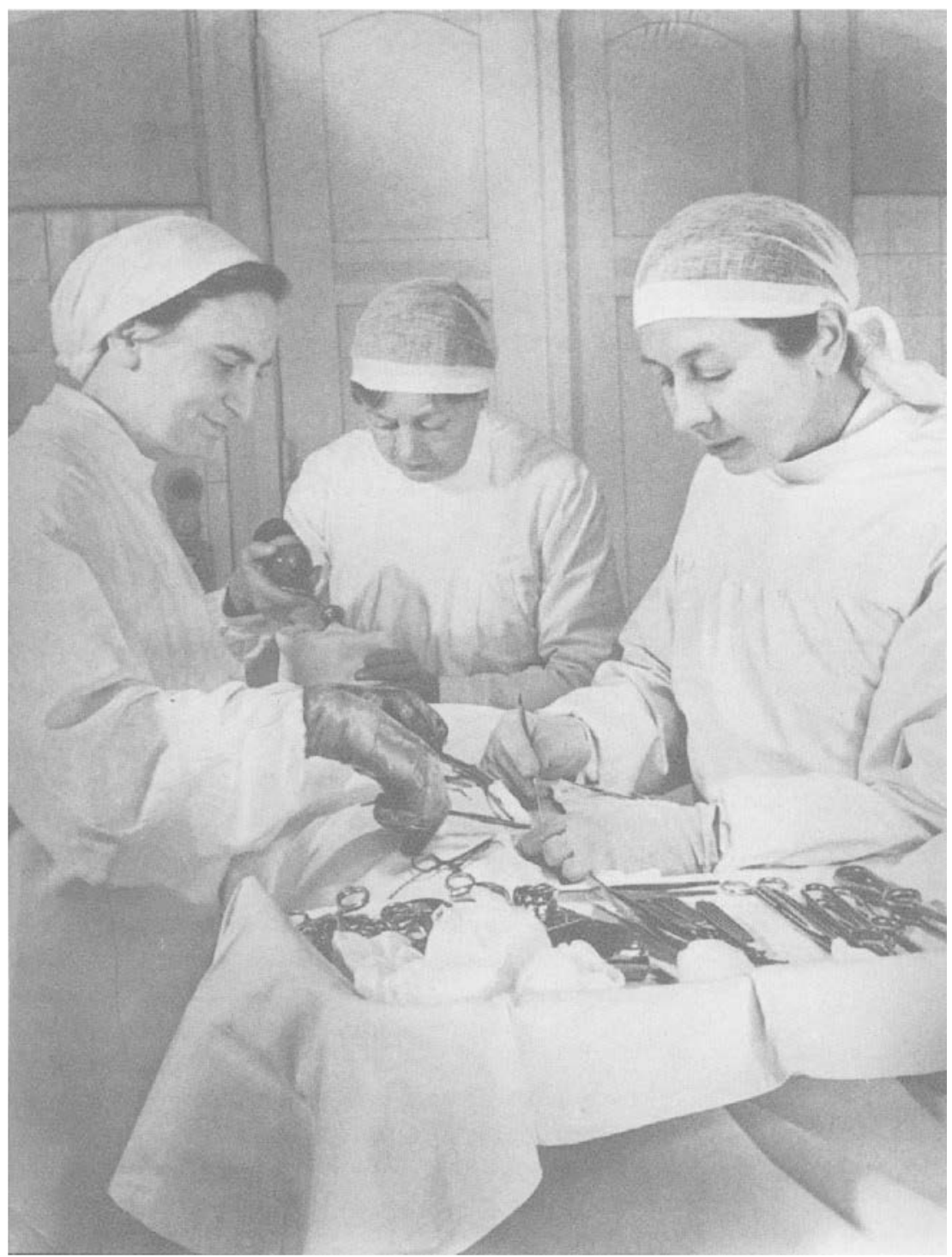

Dr. Gertrud Lullies, im Operationssaal 


\section{INHALTSVERZEICHNIS}

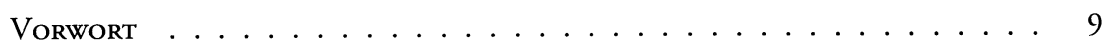

I. EINleItUng $\ldots \ldots \ldots \ldots \ldots \ldots \ldots$

II. UMfeld Und Orientierungshilfen: KLINiken Und KLINische Schulen für FraUen im AusLand

1. Internationaler Überblick . . . . . . . . . . . . . . . . . . 15

2. Die Bedeutung der Klinikgründungen für die ersten Ärztinnen . . . . . . . 28

III. Ambulante Behandlung armer Patientinnen: Die Poliklinik WEIBLICHER ÄRZTE IN BERLIN 1877-1914

1. Polikliniken und ihre Bedeutung im 19. Jahrhundert . . . . . . . . . 31

2. Die Gründung der Poliklinik weiblicher Ärzte und ihre Hintergründe . . . . 37

IV. Anfänge und Bedingungen stationärer Tätigkeit: Die Pflegeanstalt in der Friedrichstrasse . . . . . . . . . . . . 54

V. Privatwohlfahrt und Spezialisierung: Das Krankenpensionat des FräUleIN KNOOP (1894-1914)

1. Die Krankenpflegestation des Berliner Frauenverein . . . . . . . . . 71

2. Auf dem Weg zur Spezialistin: Chirurgie in der Bülowstraße . . . . . . . 85

VI. Die Chirurgische KLinik weiblicher Ärzte und die Vereinigung weiblicher Ärzte zur Gründung eines Frauenkrankenhauses in Gross-Berlin

1. Die Idee des Frauenkrankenhauses in Abgrenzung zur Privatklinik . . . . . 94

2. „Alles nur von Frauenhand ...“: Die Vereinigung weiblicher Ärzte . . . . . 95

3. „Praktische Frauenarbeit im Dienste der Wissenschaft“: Die chirurgische Privatklinik weiblicher Ärzte bis 1914 . . . . . . . . . . 102

4. Der Verein Krankenhaus weiblicher Ärzte e.V., angeschlossen an den Deutschen Lyceum-Club e.V. und die Vereinsklinik im Jahr 1914 . . . . . 112

5. Die Neue Poliklinik und das Stadtparksanatorium . . . . . . . . . . . 121

6. Das Frauenkrankenhaus als Projekt der Frauenbewegung und Ausdruck bürgerlicher Reformbestrebungen . . . . . . . . . . . . 125 
7. Diskurs über die Besonderheit der Ärztin-Patientin-Beziehung:

Engagement und Professionalisierungsstrategie . . . . . . . . . 131

8. Ausblick: Die Privatklinik weiblicher Ärzte für Frauen 1915-1933 . . . . . 137

VII. Schlussdiskussion: „Die Sondermission des weiblichen Arztes“.

Ideologie und Praxis in den Berliner Kitiniken $\ldots \ldots \ldots \ldots$

Anhang: Die Approbationsbemühungen in Deutschland tätiger

ÄrZTINNEN 1877-1908 . . . . . . . . . . . . . . . . . 156

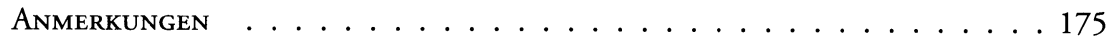

LITERATURVERZEICHNIS . . . . . . . . . . . . . . . . . . . . . . 217

Verzeichnis der Abbildungen und Faksimiles . . . . . . . . . . . 236

VERZEICHNIS DER TABELLEN $\ldots \ldots \ldots \ldots \ldots \ldots$ 


\section{VORWORT}

Dieses Buch entstand als Dissertation im Fach Medizingeschichte an der Freien Universität Berlin. Als vor zehn Jahren im Rahmen eines Colloquiums über die Wechselbeziehungen zwischen medizinischem Fortschritt, ärztlichem Selbstverständnis und Krieg auch die Frage nach der Position weiblicher Ärzte im Ersten Weltkrieg auftauchte, wurden wir gewahr, daß über die Berufsbedingungen der ersten Berliner Ärztinnen bis dato kaum Material vorlag. Weder die Lexika deutscher Ärzte noch die Standardwerke zur Medizingeschichte thematisierten den Einstieg der Frauen in die akademische Berufswelt der Ärzte. Lediglich die Autobiographien einzelner Ärztinnen wie Käthe Frankenthal, Hertha Nathorff, Franziska Tiburtius und anderer gaben Anhaltspunkte dafür, daß schon vor der Immatrikulationserlaubnis für Frauen an deutschen Universitäten in den Jahren 1902 bis 1909 eine Vielzahl akademisch gebildeter Ärztinnen im deutschen Reich praktizierten.

Hierauf begann die Bibliothekarin des Instituts, Jutta Buchin, mit der Erstellung der Dokumentation Ärztinnen im Kaiserreich, die mittlerweile über 600 Ärztinnen umfaßt. Zeitgleich entstand unter der Leitung von Frau Professor Johannna Bleker ein DoktorandInnen-Kolloquium, aus dessen Reihen mittlerweile mehrere Arbeiten zum Thema hervorgegangen sind. Ohne die über einen langen Zeitraum geleistete, geduldige und ermutigende Unterstützung von Professor Bleker, ohne die vielfachen Hinweise und Anregungen aus dem DoktorandInnenseminar, vor allem von Eva Brinkschulte, Nora Szasz, Anja Burchardt und Jutta Buchin, wäre diese Arbeit nicht zustande gekommen. Ihnen möchte ich hiermit besonders danken. Hilfreich waren auch die Hinweise der MitarbeiterInnen der Archive des Deutschen Staatsbürgerinnenverbandes, des HeleneLange-Archivs im Berliner Landesarchiv und der Bibliothek von der Zentraleinrichtung zur Föderung von Frauenstudien und Frauenforschung der FU Berlin. Für die Überlassung von Bildmaterial danke ich Oberärztin Frau Dr. Fahrig sowie dem Institut für Bibliothekswissenschaften, der Staatsbibliothek zu Berlin, Preußischer Kulturbesitz sowie dem Bundesarchiv Potsdam. Besonderen Dank schulde ich all jenen, die die Zuversicht auf das Zustandekommen dieses Buches nicht sinken ließen, als ich selber daran zweifelte: Gerhard, Daniela, Ludolf, meinen Eltern, Daniel, Leo, Katja und anderen. 